\title{
EL APRENDIZAJE COOPERATIVO COMO METODOLOGÍA PARA EL DESARROLLO DE UNA ESCUELA INCLUSIVA
}

\section{COOPERATIVE LEARNING AS A METHODOLOGY FOR INCLUSIVE EDUCATION DEVELOPMENT}

\author{
Yolanda Muñoz MARTÍNEZ1 \\ Nuria Cordero MUÑOZ ${ }^{2}$
}

RESUMEN: En este artículo se presenta la metodología del aprendizaje cooperativo como estrategia para desarrollar los principios de la educación inclusiva. Tiene una orientación muy práctica, con la intención de proporcionar herramientas para los profesores que deseen implementar esta metodología en el aula, a partir de una revisión teórica, a continuación, una descripción de un caso en el que se ha trabajado esta metodología durante 5 años. Se describen las actividades específicas y formas de trabajar con los estudiantes, finalizando con unas conclusiones sobre la aplicación de la metodología.

PALABRAS CLAVE: Aprender. Aprendizaje cooperativo. Estrategias de enseñanza. Estrategias de aprendizaje. Inclusión.

ABSTRACT: This paper presents the methodology of cooperative learning as a strategy to develop the principles of inclusive education. It has a very practical orientation, with the intention of providing tools for teachers who want to implement this methodology in the classroom, starting with a theoretical review, and then a description of a case in which they have worked this methodology for 5 years. We describe specific activities and ways of working with students, later reaching conclusions on the implementation of the methodology.

KEYWORDS: Cooperative learning. Inclusion. Learning strategies. Teaching strategies.

\section{Aprendizaje cooperativo: del individualismo a la cooperación}

En numerosos centros educativos actualmente se apuesta por un nuevo modelo de educación inclusiva frente al modelo tradicional de educación tradicionalmente segregador y excluyente. Uno de los retos que plantea el modelo educativo inclusivo es que sea el

${ }^{1}$ Universidad de Alcalá (UAH), Departamento de Ciencias de la Educación - Madrid - España. Su campo investigador y docente se basa en la Educación Inclusiva y las estrategias de mejora escolar. Ha trabajado durante 7 años en Educación Primaria y siendo parte del Equipo Directivo de un Centro Escolar. Correo: yolanda.munozm@uah.es.

${ }^{2}$ Es profesora de Educación Primaria, tiene amplia experiencia en la puesta en práctica de Aprendizaje cooperativo en las aulas, actualmente trabaja en el CEIP La Paloma de Azuqueca de Henares - Guadalajara España. Correo: nuriacm79@hotmail.com. 
profesorado de aula ordinaria, el que dé respuesta a las necesidades de todo el alumnado que forma parte de ese aula, sin excepciones. Como sostiene Martín Bris y Muñoz Martínez $(2010, \mathrm{p} .121)$

[...] dar una respuesta adecuada al contexto al que nos referimos requiere de un cambio de modelo educativo, en la actualidad basado en la homogeneidad del alumnado y su idealización, de manera que es el alumno "diferente" el que requiere medidas educativas diferenciadas, y en muchos casos excluyentes, hacia modelos que se ajusten a la individualidad de cada alumno/a, basados en la heterogeneidad y la valoración positiva de la misma, en el marco de una escuela ordinaria para Todos.

Para dotar al profesorado de herramientas para poder atender a todo el alumnado, apostamos por las metodologías de aprendizaje a través de grupos cooperativos, lo que implica profundo cambios organizativos y metodológicos.

El aprendizaje cooperativo se basa en el uso didáctico de equipos reducidos, en los que los alumnos trabajan juntos para maximizar su propio aprendizaje y el de sus compañeros de equipo. Por tanto, para comenzar a trabajar a través de equipos cooperativos, se debe partir con la creación de equipos, de composición heterogénea, que funcionen a lo largo de todo el curso escolar. El aprendizaje cooperativo se sustenta en dos pilares fundamentales según Pujolás (2004). En primer lugar, en el hecho de que el aprendizaje requiere la participación directa y activa de los estudiantes. Nadie puede aprender por otro. Como máximo puede ayudarle, pero no podrá aprender por el otro. Sólo aprendemos de verdad lo que queremos aprender y siempre que participemos activamente en el proceso de aprendizaje, de ahí la importancia de la motivación y del aprendizaje participante.

Se trata de evitar que en el aula haya un grupo de sujetos que protagonice casi todas las interacciones y éxitos, así como otro grupo de sujetos que no intervenga casi nunca ni consiga el más mínimo éxito o reconocimiento académico. Así pues, los alumnos más expertos, capaces de resolver la tarea por ellos mismos, aprenden a enseñar a otros compañeros más "novatos" integrando de esta manera el objetivo de la tarea y mejorando otras habilidades lingüísticas y sociales; y a su vez el compañero novato recibe la explicación de la tarea relacionada con su contexto inmediato, intereses ya que quien le aclara los objetivos es un igual.

En los contextos en los que además conviven diversos grupos étnicos o culturales contribuye a desarrollar la tolerancia cuando se dan oportunidades de igualdad de estatus que permitan establecer relaciones de amistad con miembros de otros grupos; y promueven la 
tolerancia y luchar contra la exclusión. Los miembros de un equipo de trabajo cooperativos tienen una doble responsabilidad: aprender ellos lo que el profesor les enseña y contribuir también a que lo aprendan sus compañeros de equipo, como un contenido escolar más.

Es una metodología activa, basada en la experiencia e interacción entre los alumnos y que posibilita que los alumnos aprendan unos de otros, así como del entorno y del profesor, siendo el rol de éste último la supervisión activa y no directiva tanto del proceso de aprendizaje, como de las interacciones entre los alumnos.

Numerosas investigaciones destacan sus ventajas, entre las que podemos encontrar las siguientes:

- $\quad$ El aprendizaje cooperativo constituye una opción metodológica que valora positivamente la diferencia, la diversidad de niveles de desempeño, de culturas de origen, de capacidades, circunstancia que tradicionalmente se ha visto como un inconveniente, se convierte en un recurso de aprendizaje.

- Contribuye al desarrollo cognitivo: favorece la asimilación de nuevas estrategias para aprender, a partir de la observación de otros modelos. Los alumnos consiguen importantes avances en el desarrollo cognitivo, independientemente de su nivel, ya que los alumnos interactúan y se apoyan independientemente de si son más o menos aventajados.

- $\quad$ Reduce la ansiedad: contribuye a reducir la ansiedad en la medida que fomenta la autoestima de los alumnos y la confianza en sí mismos, ya que les permite que se relajen y trabajen en un entorno tranquilo y adecuado para pensar, ensayar y recibir retroalimentación tanto del profesor como de sus compañeros, derivándose de ello mayores posibilidades de éxito.

- Fomenta la interacción: produciéndose una interacción constante entre los estudiantes, lo que fomenta el desarrollo intelectual y propiciando situaciones de enseñanza y aprendizaje que superan los modelos individualistas.

- Fomenta la autonomía y autorregulación en el aprendizaje: se reduce considerablemente la dependencia de los alumnos con respecto al profesor, ya que los compañeros pueden proporcionar el tipo de apoyo que antes corría a cargo del profesor. Permite que los alumnos e impliquen en una variedad de procedimientos que antes estaban reservados al profesor, derivando en una mayor responsabilidad para los alumnos (explicar, supervisar, corregir, etc).

- Adecua los contenidos al nivel de los alumnos: la interacción en grupo facilita la comprensión por parte de los alumnos de los contenidos curriculares. El trabajo cooperativo produce una seguridad en los alumnos que acaba fomentando la participación de los más tímidos e inseguros en el trabajo conjunto y la distribución equitativa del turno de palabra.

- Desarrolla el pensamiento crítico: cuando los alumnos trabajan en contextos cooperativos, se ponen en juego toda una serie de destrezas metacognitivas relacionadas con 
la propia interacción cooperativa: planificación y organización de la tarea, toma de decisiones, argumentación y defensa de posturas, negociación de puntos de vista, resolución de problemas.

- Favorece los procesos de inclusión educativa: las dinámicas cooperativas contribuya a:

-Compensar situaciones de exclusión social, sobre todo en el caso de alumnos rechazados.

-Promover relaciones multiculturales positivas, reforzando los vínculos entre alumnos que proceden de diferentes etnias, grupos sociales y culturales.

-Mejorar la inclusión de estudiantes con necesidades educativas especiales.

- Favorece el desarrollo socio-afectivo: contribuye significativamente al aumento de la cantidad y calidad de las interacciones entre los alumnos, lo que fomenta el desarrollo de habilidades sociales y comunicativas y habilidades para el trabajo en grupo. Esta interacción constante se traduce en una mayor cohesión dentro del grupo-clase.

- Aumenta la motivación hacia el aprendizaje escolar: La metodología cooperativa incide positivamente sobre algunas de las variables fundamentales relacionadas con la motivación hacia el aprendizaje escolar:

- Probabilidad subjetiva de éxito y atribución causal;

- Curiosidad epistémico y motivación continuada;

- Compromiso con el aprendizaje;

- Persistencia en la tarea;

- Expectativas de éxito futuro y nivel de aspiración.

- Mejora el rendimiento académico: Las investigaciones demuestran que los alumnos aprenden más y mejor en contextos cooperativos que en aquellas aulas presididas por un clima competitivo o individualista. Esta constatación se hace extensible a todas las áreas, sea cual sea el nivel de rendimiento que presenten los alumnos.

- $\quad$ Contribuye a reducir la violencia en la escuela: El aprendizaje cooperativo constituye una herramienta para reducir algunos de los factores más decisivos en la aparición de comportamientos violentos, como pueden ser:

- El fracaso escolar.

- Falta de vínculos con sus compañeros.

- Trastornos psicopedagógicos.

Por lo tanto podemos decir que la base de esta metodología es fomentar la cooperación y solidaridad y compensación de la desigualdad, ya que se legitiman las 
conductas de pedir y proporcionar ayuda, mejorando con ello tanto el repertorio social de los alumnos como sus oportunidades de aprendizaje. Los niños suelen ser con frecuencia receptores de la ayuda de los adultos. Muy pocas veces tienen la oportunidad de comprobar su propia eficacia ayudando a otra persona y de mejorar su autoestima y sentido de autoeficacia.

\section{El comienzo del trabajo: cuestiones de partida}

Con el objetivo de facilitar la puesta en marcha de una nueva metodología de enseñanza y aprendizaje, que además conlleva la creación de materiales y una exhaustiva programación de actividades, hay que mejorar la organización de aula, en la que participaran profesores con una actitud positiva hacia nuevas metodologías y con predisposición al cambio, teniéndose también en cuenta el perfil profesional de ellos. Observándose con ello que es fundamental el apoyo del equipo directivo en el que se quiera instaurar esta metodología.

Es básico, estudiar de forma más exhaustiva la diversidad del aula, diseñar actividades que estimulen la construcción del conocimiento ( internet en el aula, uso de ordenadores, murales, etc), enseñar a cooperar y resolver conflictos de forma positiva, observar lo que sucede en los grupos, distribuir las oportunidades de protagonismo dentro del mismo, etc.

Es importante estructurar bien la tarea y el trabajo cooperativo, para que resulte más fácil. La función de los profesores durante la realización de las actividades será de supervisión y orientación, comprobando el proceso de ejecución de la tarea, las interacciones entre los miembros del equipo, el reparto de funciones que han hecho en el mismo pudiendo ayudarles a cambiar alguna función según el rendimiento de los alumnos.

Para crear un clima de trabajo de grupo adecuado, y tendente a la cooperación, se anima a los alumnos a ayudar a los otros miembros de su equipo en la realización de la tarea encomendada. Hay que transmitir al alumnado la idea de que se recompensa por el rendimiento obtenido como consecuencia del trabajo a todo el grupo, y que es necesaria la participación de todos los miembros del grupo para obtener dicha recompensa.

De lo anteriormente expuesto se deriva que las funciones del profesor, en el modelo de enseñanza cooperativo, se alejen sustancialmente del perfil de profesor que el modelo tradicional de enseñanza defiende. En el aprendizaje cooperativo el profesor se considera un facilitador del aprendizaje, gestionando y coordinando el trabajo de los equipos, alguien que 
orienta a la hora de encontrar la respuesta a los problemas, que da pistas para que los alumnos investiguen, etc; un mediador del aprendizaje, no un transmisor de conceptos.

\section{La organización espacial del aula}

A continuación se presenta una experiencia de aprendizaje cooperativo en Educación Primaria, que se lleva a cabo desde hace varios años en el CEIP La Paloma, para poder explicar, desde un punto de vista práctico en qué consiste y cómo se organiza un clase real.

La clase se divide en equipos de aprendizaje de 4 y 5 miembros, cuidando que éstos sean heterogéneos en rendimiento y género los cuales permanecerán estables a lo largo de todo el curso, aunque pueden variar sus miembros, siempre a criterio del profesorado del aula. Para la organización de los equipos se deben tener en cuenta diversos factores, precisamente para respetar el criterio de heterogeneidad, fundamental en esta forma de aprendizaje, y fundamental también en lo que entendemos como una escuela inclusiva. De ahí que los equipos deben estar formados por estudiantes que sean diferentes en cuanto a:

- $\quad$ Rendimiento académico.

- $\quad$ Nivel de solidaridad.

- $\quad$ Nivel de tolerancia ante las desigualdades.

- $\quad$ Nivel de rechazo o aceptación entre los compañeros/as.

- Sexo.

Como se deriva de lo anterior, los integrantes de los equipos son seleccionados de forma intencional, por parte del profesor, teniendo en cuenta que una de las ideas básicas de esta forma de trabajo es que los alumnos trabajen juntos para la consecución de metas comunes, beneficiosas para todos.

La organización del aula favorecerá el trabajo por equipos, así pues las mesas estarán dispuestas para poder trabajar en grupo y de forma que todos los componentes puedan mirar la pizarra sin dificultad.

En ocasiones no se trabaja en equipo siendo los alumnos los que organizan sus mesas de manera que quedan dispuestas en grupos o no, y son ellos mismos los que se cambian de sitio, al principio tardan mucho en colocar la clase, pero poco a poco van mejorando, hasta que consiguen hacerlo de forma ordenada y sin ruido, evitando arrastrar las mesas y sillas. 


\section{Organización y funcionamiento de los equipos}

Los estudiantes del aula, como se ha comentado anteriormente, están organizados en grupos, de cuatro o cinco alumnos, en los cuales cada uno cumple una función concreta (rol) asignada, y que puede variar en función de la edad, tarea, estructura del aula, etc.

- Coordinador: Su función es controlar el trabajo del grupo y su buen funcionamiento.

- Secretario: Rellena las hojas de control del equipo y toma notas de todo aquello que deba estar plasmado en un papel.

- Portavoz: Es la voz del grupo ante la clase y el profesor.

- Mediador: Es el encargado de solucionar los conflictos que puedan surgir en el grupo, y de alentar a sus compañeros.

- Material y orden: Se encarga de repartir y cuidar el material del grupo, y de mantener todo bien ordenado.

Los propios alumnos, al comienzo de curso, se dictan sus propias normas de funcionamiento, en base a las cuales tendrán que evaluar el comportamiento de sus compañeros, e incluso el de sí mismos. Tras haber trabajado en las normas generales de grupo-clase, los alumnos deben empezar a crear sus propias normas de grupo e individuales.

Imagen 1: Normas realizadas por los propios estudiantes

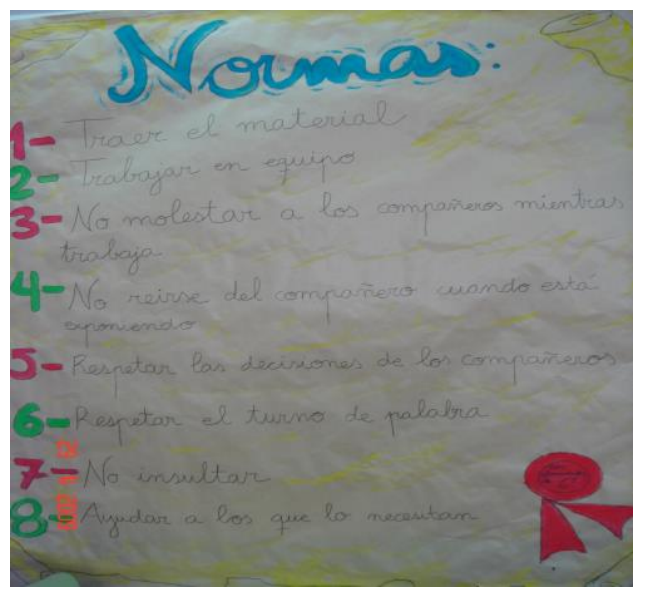

Fuente: Archivo de la investigación.

Es una metodología que tiene mucho en cuenta las opiniones de los alumnos. Otra prueba de ello es el "Plan de equipo" que realizan, para llevar un registro de sus objetivos y compromisos personales dentro del grupo. 
Imagen 2: Estudiantes trabajando en los equipos

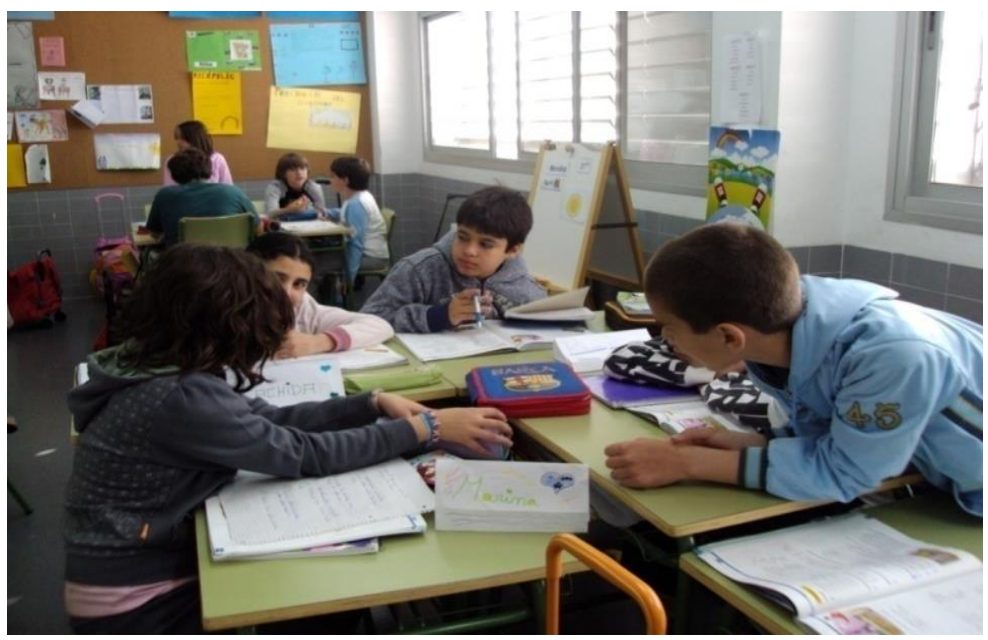

Fuente: Archivo de la investigación.

A principio de curso se marcan unos objetivos a conseguir, y ellos mismos, con el paso del tiempo y según su evolución deben adquirir compromisos tanto con su grupo como con la profesora, para mejorar. Es una forma también de darles a los niños la ocasión de sentirse más responsables, de crear cierto peso de cada uno en el grupo. De reforzar autoestima, en definitiva.

Por otra parte, los propios integrantes del grupo deben mantener el orden en el mismo, y evitar los conflictos entre ellos. Aunque es un tanto difícil. Por eso el mediador registra el conflicto (con fecha, firma de los implicados, solución propuesta....) en una hoja destinada a ello, y se lo hace llegar al profesor.

Además, al final de cada trimestre, el profesorado les pasa un cuestionario a rellenar en grupo, en el cual deben reflexionar sobre el trabajo realizado. Deben evaluar y, sobre todo, justificar su trabajo o su falta de ello, tanto de forma individual, como grupal.

La evaluación en grupos cooperativos difiere en varios aspectos respecto de la evaluación tradicional que se basa únicamente en el examen escrito. Así pues, la evaluación "cooperativa" valora: el trabajo individual de cada miembro dentro del equipo, la actuación del propio grupo cooperativo, promueve la autoevaluación y la coevaluación.

De esta forma, la calificación de cada alumno consiste en: la puntuación obtenida en la prueba individual de conocimientos; la valoración de las profesoras sobre el funcionamiento del equipo cooperativo y la autoevaluación del equipo al finalizar el trimestre, siendo también importantes los trabajos y cuadernos de forma individual. El funcionamiento del equipo se revisará periódicamente y se evaluarán finalmente sus logros. 
Por otra parte, son los propios alumnos quienes evalúan el trabajo del resto de la clase (co-evaluación), a través de una plantilla de coevaluación que el profesor facilita. En el caso de exposiciones, murales, cuentos, etc; para ello se les facilita una tabla con los criterios que deben evaluar. Las notas de cada uno de los grupos hacen media con la nota del profesor.

Además, esta forma de trabajo está íntimamente ligada a un refuerzo positivo continuo, para motivar e incentivar a los alumnos. Las dificultades que entraña la motivación a alumnos que provienen de diferentes contextos vulnerables, se intentan superar a través de herramientas como: tarjetas de tema, tarjetas-imán de fin de trimestre, y sobre todo, hay que hacer hincapié en el refuerzo positivo que reciben los niños del profesorado. Siendo fundamental compaginar muy bien el refuerzo académico, con dicho refuerzo. Estas tarjetas, son repartidas en función de lo productivo que haya sido, o no, el desarrollo del tema, y de los resultados globales obtenidos en la evaluación del tema.

\section{Optimizando los recursos personales para atender a la diversidad}

Como hemos dicho anteriormente, los modelos inclusivos en educación abogan por atender al alumnado dentro de su propia aula, sin tener que ir a otros espacios, como ocurría anteriormente con el modelo de Educación Especial. Gracias a esta metodología se reduce profesorado, por ejemplo para la atención de alumnos extranjeros sin idioma (como nos ha demostrado la experiencia durante cinco años con numerosos estudiantes de diferentes nacionalidades), ya que el estar en comunicación continua con sus compañeros el aprendizaje de un nuevo idioma se realiza sin tantas dificultades y en menor tiempo.

En el caso de alumnado con necesidades educativas especiales ocurre lo mismo, ya que la atención que recibe, a través de los grupos cooperativos, es más amplia, así como su participación, llegando en muchas ocasiones a poder prescindir, como nos ha demostrado la experiencia, de la atención de otros especialistas que normalmente les ofrecen refuerzo y apoyo educativo.

\section{Tareas cooperativas: algunos ejemplos de actividades de aula}

Las características de las tareas desarrolladas, deben ser, principalmente atractivas, con una recompensa al esfuerzo y que se resuelvan siempre mediante la intervención de cada uno de sus miembros. Algunas de estas tareas son:

- Actividades de fichas colectivas sobre cuestiones relativas a los contenidos trabajados y que deben ser resueltas en equipo. 
- Realización de murales sobre los temas trabajados que después deberán ser expuestos en voz alta para todo el aula.

- Trabajos de investigación en Internet con el ordenador portátil dentro del aula y en el

- $\quad$ aula de ordenadores del Centro Educativo.

- Webquests y el blog creado para cada una de las áreas.

- $\quad$ Elaboración de preguntas en grupo sobre el temario que se está estudiando.

- Wikis en las que los diferentes equipos preparan y resuelven cuestiones elaboradas por otros compañeros y en las que elaboran trabajos.

- Juego-concurso con las preguntas que los alumnos/as han elaborado anteriormente

- Exposiciones orales de los trabajos realizados, tanto a la clase como a niveles inferiores.

- Transformar a los alumnos en profesores, tarea de la que se hablará posteriormente por la complejidad que supone.

\section{El uso de las nuevas tecnologías y el blog como estrategia cooperativa}

Actualmente las nuevas tecnologías han tomado fuerza en nuestra sociedad y es fundamental que los alumnos conozcan cómo funcionan, la ayuda y facilidad que nos proporcionan en la realización de diferentes tareas, pero ante todo es básico que los alumnos sepan hacer un uso correcto de éstas. Para ello es necesario un dominio básico de dichas herramientas por parte del profesorado, por lo que es fundamental desarrollar programas de formación de profesores, como sostiene Díaz-Aguado (2003), para quien la formación de los profesores es una herramienta clave para superar las dificultades, facilitando dicha formación la adquisición de recursos eficaces para adaptar la educación a la diversidad de los alumnos, a la cooperación entre profesores, así como la participación e implicación de la Comunidad Educativa.

Recurriendo a ellas (pizarra digital, ordenadores portátiles, uso de internet, wikis, blogs) se ha observado un alto nivel de motivación en los alumnos, aprenden a buscar información en distintas fuentes, más allá del libro de texto o de la explicación del profesor. A lo largo de los últimos dos años, los estudiantes han comprobado que pueden usar las tecnologías para facilitar el estudio y darles un buen uso. Los alumnos han mejorado todas las 
competencias, incluyendo la competencia digital, han ganado en autonomía y confianza y asimilan con más claridad los contenidos a trabajar.

$\mathrm{Su}$ uso ha hecho que las exposiciones de trabajos sean más amenas y motivadoras para ellos, ya que pueden acceder a más fuentes y soportes, como Power Point o Cuadernia, este último software creado en Castilla La Mancha y que tiene muchas facilidades para alumnos de menores edades.

Imagen 3: Estudiante explicando contenidos a sus compañeros en la pizarra digital

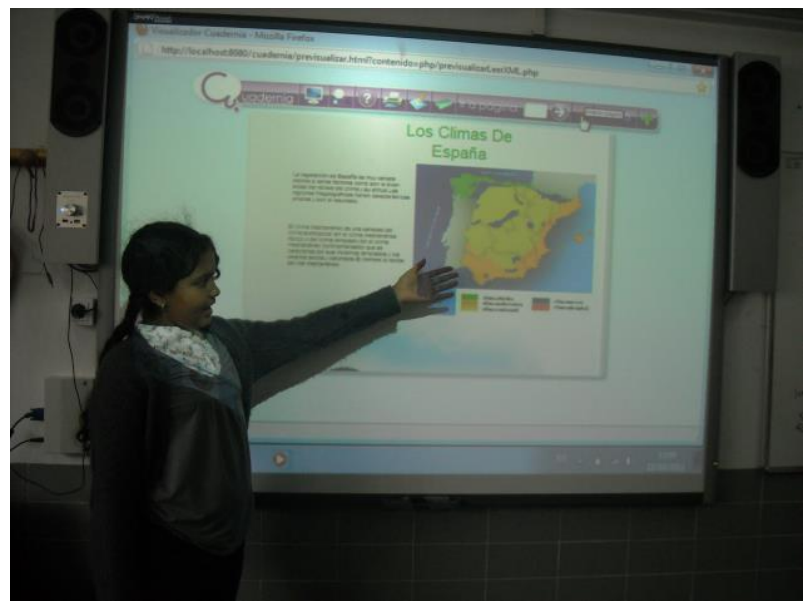

Fuente: Archivo de la investigación.

Así mismo, habitualmente, acceden al blog del colegio, en el que se han creado enlaces a las diferentes áreas, por cursos, gracias a los cuales pueden ampliar información, buscar material para sus exposiciones, consultar dudas con los compañeros.... En ellos también encuentran accesos a wikis, organizadas por equipos en las que plasmas sus trabajos e incluso plantean cuestiones para el resto de los equipos.

Imagen 4: Blog creado por la profesora, y usado por los estudiantes y familias

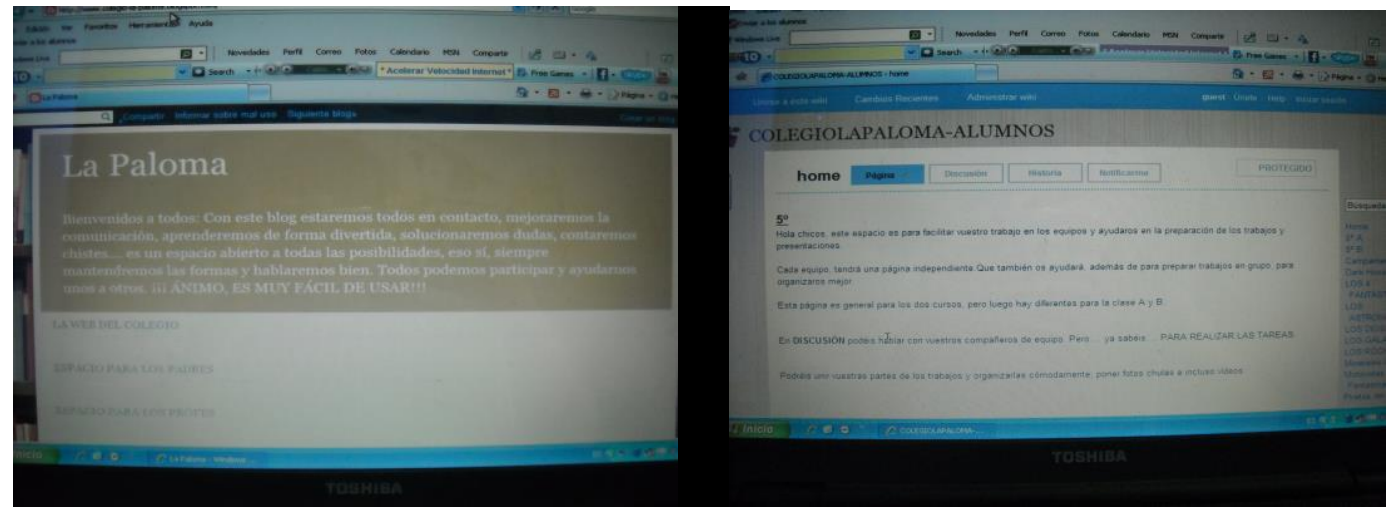

Fuente: Archivo de la investigación. 


\section{Un paso más ante el trabajo a través de grupos cooperativos}

Tras analizar los beneficios de esta metodología y que los alumnos asumían perfectamente; hemos adoptado una nueva forma de trabajo, siempre basada en el aprendizaje mediante grupos cooperativos, que es transformar a los alumnos en profesores.

Dicha forma de trabajo se ha llevado a cabo en las áreas de Matemáticas y Lengua, ya que consideramos (las dos profesoras implicadas) que podía ofrecer un resultado muy positivo por diversos motivos:

Los alumnos aprenderían a buscar información a través de diferentes vías (biblioteca, material del profesor, internet...) y seleccionar la más relevante para llevar a cabo su tarea educativa y plasmar la teoría de la sección que a cada grupo le correspondiera.

Así mismo, se han inventado problemas y actividades, basados en los propuestos por las diversas editoriales, lo que ha mejorado su capacidad de razonamiento, expresión y creatividad.

Observábamos la necesidad de que los alumnos sintieran la labor que realiza un docente, con lo que mejoraría la visión de estos hacia el profesorado; así como vivir lo importante que es el respeto profesor-alumno y viceversa.

La clase contaría con el refuerzo de más profesores ( ya que al estar cada grupo compuesto por 4 o 5 componentes, el número de maestros en el aula también era mayor) lo que beneficiaría a la hora de atender las cuestiones que siempre surgen en una clase.... solucionar dudas, mantener el orden y promover que los alumnos que se distrajeran con facilidad, se mantuvieran centrados.

Aumentaría la autoestima y por consiguiente aquellos compañeros con más problemática para expresarse en clase se animarían a ello, con la seguridad de dominar los temas que van a tratar.

La organización de esta tarea cooperativa se realiza de la siguiente forma:

Previamente, las profesoras seleccionábamos la parte del tema que iba a abordar cada grupo y se les informaba de ello. Comenzando, desde este momento, la preparación de la clase y su labor como maestros. Esta parte es fundamental, ya que todos los compañeros tienen que tener claros los contenidos, para poder continuar con la tarea. Por lo que entre todos comentarán y solucionarán las posibles dudas que puede tener algún miembro del grupo.

Posteriormente, con la ayuda de los coordinadores, se analizarán las cuestiones y contenidos a abordar y se planteará un debate para analizar la mejor forma de llevar a cabo la tarea de explicar.

Tras este paso, el coordinador reparte el trabajo que realizará cada miembro (búsqueda de información en libros u ordenador para preparar la teoría a explicar al resto de la clase, preparación de actividades o fichas, búsqueda de páginas web para que los compañeros 
acudan al aula de ordenadores), las opciones son infinitas, ya que se les da libertad de movimiento, para lo que es fundamental, mantener orden, respeto y un volumen adecuado al hablar, labor que vigila, como hemos comentado anteriormente el mediador del equipo.

El secretario, recogerá en el documento de equipo, la labor encomendada a cada componente y que posteriormente se evaluará y valorará.

El encargado del material y orden, facilitará a sus compañeros el material que les pudiera ser necesario (cartulinas, folios, témperas...) y será encargado de hacer las fotocopias que fueran oportunas...siempre pensando en el cuidado a la naturaleza y no malgastando folios...intentando siempre recurrir a la pizarra o al dictado.

Los portavoces, transmitirán al profesor, como la voz del grupo que es, posibles problemáticas, situaciones o dudas que el grupo por sí mismo no pueda solucionar.

Como se observa, todos los componentes, a la vez que preparan su parte, deben cumplir con los roles otorgados por trabajar en grupo cooperativo, por lo que se mantiene la filosofía siempre la estructura fijada desde comienzo de curso.

La preparación de sus clases durará dos o tres días, dependiendo del tema que se esté trabajando y posteriormente....cada grupo, en días sucesivos irán dando sus clases, con todo lo que ellos conlleva: explicación, atención al comportamiento, corrección de actividades por parte del resto de los compañeros del aula....

Al finalizar cada una de las sesiones, el resto del grupo valorará cómo se ha llevado a cabo la labor docente, gracias a los documentos que proporcionamos previamente a todos los grupos.

Finalmente, se realiza una prueba para analizar la consecución de los objetivos planteados, dicha prueba recoge todos los contenidos que han abordado todos los grupos. Y estos completan sus documentos, tanto la coevaluación de su trabajo como grupo como la autoevaluación de cada uno de los miembros.

La evaluación final del tema y su calificación se hará teniendo en cuenta los siguientes aspectos, que ellos conocen de antemano: cómo han preparado y llevado la clase, las autoevaluaciones y coevaluaciones y las pruebas escritas.

\section{Conclusión}

Los resultados obtenidos de esta metodología han sido muy positivos por los siguientes motivos:

- Los alumnos han mejorado su comportamiento y actitud en el aula. 
- Han mejorado su punto de vista sobre la labor del maestro.

- Ha resultado muy beneficioso de cara a la autonomía.

- Han aprendido a buscar información a través de diferentes vías.

- Hacen las tareas encomendadas gustosamente, sin plantear quejas.

- La limpieza de los trabajos y cuadernos ha mejorado ya que, como el resto de los compañeros ven éstos, se esfuerzan por hacerlos con una mejor presentación.

- El respeto y las relaciones entre los alumnos del aula ha cambiado, siendo más intensas y respetuosas.

- Asimilan con mucha más claridad los contenidos a trabajar.

- Todos los miembros participan en las actividades, incluyendo los que con anterioridad se mostraban reticentes.

\section{REFERENCIAS}

DIAZ-AGUADO, M. J. Educación intercultural y aprendizaje cooperativo. Madrid: Pirámide, 2003.

MARTÍN BRIS, M.; MUÑOZ MARTÍNEZ, Y. La participación del profesorado en un proceso de mejora en el marco de una escuela para todos. Revista Iberoamericana sobre Calidad, Eficacia y Cambio en Educación, Madrid, v.8, n.3, p.121-138, 2010.

PUJOLÁS, P. Aprender juntos, alumnos diferentes. Barcelona: Octaedro, 2004.

\section{Como referenciar este artigo}

MARTÍNEZ, Yolanda Muñoz; MUÑOZ, Nuria Cordero. El aprendizaje cooperativo como metodología para el desarrollo de una escuela inclusiva. Doxa: Rev. Bras. Psicol. Educ., Araraquara, v.19, n.1, p. 149-162, jan./jun. 2017. ISSN: 1413-2060.

Submetido em: 20/01/2017

Aprovado em: 30/03/2017 